\title{
Nutrient supplementation modulates angiotensin II-mediated atherosclerosis in ApoE KO mice
}

\author{
VADIM IVANOV, JOHN CHA, SVETLANA IVANOVA, TATIANA KALINOVSKY, \\ MATTHIAS RATH and ALEKSANDRA NIEDZWIECKI \\ Dr. Rath Research Institute, Santa Clara, CA 95050, USA
}

Received March 1, 2010; Accepted March 24, 2010

DOI: $10.3892 / \mathrm{mmr} 00000274$

\begin{abstract}
We studied the effect of nutrient/herbal extract mixture (NM) supplementation prior to angiotensin II (AngII) administration on ApoE KO mice. AngII-mediated injuries, which included increased atherosclerotic lesion size (by $31 \%$ ) and severity (by 200\%), as well as an increased number of necrotic cores (by 44\%), were attenuated by NM supplementation, resulting in the reduction of lesion size by $20 \%(\mathrm{p}=0.09)$, lesion severity by $31 \%(\mathrm{p}=0.13)$ and the average number of necrotic cores per arterial tree by $39 \%(\mathrm{p}=0.35)$, compared to mice fed the regular diet. The frequency of Type B thoracic aortic dissections and abdominal aneurysms in AngII-treated mice was reduced from $55.6 \%$ to 14.3 and $42.9 \%$, respectively, with NM supplementation. Macrophage density in the aortic lesions of NM-supplemented AngII-treated mice was $20 \%$ lower $(\mathrm{p}=0.033$ ) than in mice on the regular diet, whereas collagen density in NM mice was increased by $208 \%$ $(\mathrm{p}=0.026)$ compared to that in mice fed the regular diet, making for a more stable plaque type. NM-supplemented mice showed a significant reduction in total cholesterol $(32 \%$ decrease, $\mathrm{p}=0.0001)$ and LDL (66\% decrease, $\mathrm{p}<0.0001)$, commonly measured risk factors for cardiovascular disease. In conclusion, NM consumption prior to AngII administration in ApoE KO mice minimized aortic dissections, inflammatory monocyte recruitment to plaques, plaque mass and abdominal aortic dilation.
\end{abstract}

\section{Introduction}

Numerous clinical and epidemiological studies have identified systemic arterial hypertension as an independent and potent risk factor for the development of atherosclerosis $(1,2)$. Studies have implicated the renin-angiotensin II system in

Correspondence to: Dr Aleksandra Niedzwiecki, Dr. Rath Research Institute, 1260 Memorex Drive, Santa Clara, CA 95050, USA

E-mail: author@drrath.com

Key words: atherosclerosis, angiotensin II, ApoE KO mice, aortic dissection atherogenesis. Angiotensin II (AngII) is known to mediate its atherogenic effects through superoxide radical formation, cytokine generation by the arterial wall and mechanical stresses that result from vasoconstriction. Genetically engineered mouse models, such as ApoE KO mice, have been developed to help elucidate the pathogenesis of atherosclerosis $(3,4)$. AngII has been shown to intensify atherosclerosis and to generate aortic dissections in mice $(5,6)$.

Epidemiological studies have shown that the high consumption of fruits and vegetables is associated with a reduced risk of cardiovascular disease (7). Clinical studies have demonstrated that the consumption of fruits and vegetables for several weeks or months reduced such parameters as cholesterolemia, oxidative stress, homocysteinemia, endothelial dysfunction and blood pressure (8-11). Flavonol intake (mainly apple and tea) was inversely correlated to coronary mortality in women (12). Furthermore, epidemiologic and clinical studies have documented the benefits of individual nutrients in the prevention of cardiovascular disease $(13,14)$. Our study confirmed the cardioprotective effects of supplementation with vitamins, minerals, amino acids and co-enzymes on the effects of nutrient supplementation on the progression of early coronary atherosclerosis. In this pilot study, the extent of coronary calcification in 55 patients diagnosed with early coronary atherosclerosis was measured prior to and after 1 year of nutrient supplementation using an Imatron C-100 Ultrafast CT scanner. Progression of coronary calcification as determined by the coronary artery scan score decreased significantly (from 0.49 to $0.28 \mathrm{~mm}$ monthly growth) after a year of nutritional intervention (15).

Naturally occurring compounds demonstrate a wider spectrum of biological activity and fewer side effects than synthetic drugs, and a mixture of natural compounds often produces synergistically enhanced therapeutic actions. This reasoning prompted us to investigate whether nutrient and herb supplementation of ApoE (-/-) mice would beneficially modulate AngII-induced atherosclerotic damage. The specific nutrient/herb mixture (NM) was formulated based on the anti-atherogenic effects of the compounds. For example, apoprotein E-deficient mice fed green tea for 14 weeks exhibited an attenuation of aortic atheromatous areas by $23 \%$ and decreased aortic cholesterol and triglyceride levels compared to the control group (16). 
Table I. Treatment summary.

\begin{tabular}{lcccc}
\hline $\begin{array}{l}\text { Groups } \\
\text { (no. in group) }\end{array}$ & $\begin{array}{c}\text { Murine } \\
\text { genotype }\end{array}$ & $\begin{array}{c}\text { Diet duration (12 weeks } \\
\text { starting at 24 weeks of age) }\end{array}$ & $\begin{array}{c}\text { AngII treatment osmotic pump } \\
\text { duration (age at implantation) }\end{array}$ & $\begin{array}{c}\text { Age at time of harvest } \\
\text { (weeks) }\end{array}$ \\
\hline $1(n=9)$ & ApoE KO & Regular rodent diet & None & 24 \\
$2(n=9)$ & ApoE KO & Regular rodent diet & None & 36 \\
$3(n=9)$ & ApoE KO & Regular rodent diet & 4 weeks (32 weeks) & 36 \\
$4(n=9)$ & ApoE KO & Supplemented NM 0.5\% & None & 36 \\
$5(n=7)$ & ApoE KO & Supplemented NM 0.5\% & 4 weeks (32 weeks) & 36 \\
$6(n=8)$ & Wild-type & Regular rodent diet & None & 24 \\
$7(n=9)$ & Wild-type & Regular rodent diet & None & 36 \\
\hline
\end{tabular}

\section{Materials and methods}

Diet. The regular and supplemented mouse chow (0.5\% NM w/w) were milled and pressed by Purina Mills, LLC. NM was prepared by VitaTech (Hayward, CA) and consisted of the following ingredients in the relative amounts indicated per $1000 \mathrm{mg}$ NM: $161 \mathrm{mg}$ green tea extract (40\% EGCG); $113 \mathrm{mg}$ gotu kola extract (10\% asiatic acid); $81 \mathrm{mg}$ quercetin; $194 \mathrm{mg}$ L-ascorbic acid; $161 \mathrm{mg}$ L-lysine; $161 \mathrm{mg}$ L-proline; $32 \mathrm{mg}$ L-methionine; $32 \mathrm{mg}$ L-cysteine HCL; $58 \mathrm{mg}$ choline bitartrate; $1.7 \mathrm{mg}$ copper glycinate; $3.2 \mu \mathrm{g}$ cyanocobalamin; $0.2 \mathrm{mg}$ folic acid and $1.3 \mathrm{mg}$ pyridoxine $\mathrm{HCl}$. Mice consumed approximately $4 \mathrm{~g}$ of chow per day (measured as an average of food weight consumed by 36 mice over $\sim 3$ weeks); thus, supplemented mice consumed $\sim 20 \mathrm{mg}$ of NM per day or $800 \mathrm{mg} / \mathrm{kg}$ bodyweight.

Animals. Eight-week-old C57B6 ApoE (-/-) male mice ( $\mathrm{n}=45)$ and wild-type male mice $(\mathrm{n}=17)$ were obtained from Jackson Laboratories. Mice were acclimatized and maintained on a 12-h light/dark cycle with provision of Purina Test Diet mouse chow without additives and water ad libitum in pathogen-free microinsulator cages. All procedures were performed according to the humane and customary care and use of experimental animals, and followed a protocol approved by the Internal Animal Care and Use Committee of the Dr. Rath Research Institute.

Treatment groups. The mice were divided into groups as follows: the 45 ApoE KO mice were divided into 5 groups of 9 mice each (Groups 1-5). Group 1 (baseline control) mice were fed a diet not supplemented with AngII and were harvested at 24 weeks of age. Group 2 mice were maintained on a regular rodent diet not supplemented with AngII and were harvested at 36 weeks of age. Group 3 mice were maintained on a regular rodent diet, implanted with AngII-loaded osmotic pumps (Alzet model 2004; ALZA, Palo Alto) at 32 weeks of age and harvested 4 weeks later at 36 weeks of age. Group 4 mice were maintained on $0.5 \%$ NM chow for 12 weeks and were harvested at 36 weeks of age. Group 5 mice were maintained on $0.5 \%$ NM chow for 12 weeks, implanted with AngII-loaded osmotic pumps at 32 weeks of age and harvested at 36 weeks of age. Two animals succumbed to complications due to surgery. The 17 wild-type mice, which served as the controls, were divided into two groups: Group $6(n=8)$ mice, harvested at 24 weeks of age, and Group $7(n=9)$ mice, harvested at 36 weeks of age. A summary of the treatment groups is shown in Table I.
Angiotensin II osmotic pump installation. Using an anaesthesia machine (VMS Matrx) with a precision isoflurane vaporizer and pressurized oxygen gas, 18 ApoE (-/-) mice were induced before being implanted with osmotic pumps (Alzet Model 2004) in the dorsum subcutaneous pouch. Each pump contained $200 \mu \mathrm{l}$ of $4 \mathrm{mM}$ human AngII, $150 \mathrm{mM} \mathrm{NaCl}$ and $0.01 \mathrm{~N}$ acetic acid (previously sterilized by $0.2 \mu \mathrm{M}$ filtration). At these conditions, AngII was released at an approximate rate of $27 \mathrm{nmol}$ per $24 \mathrm{~h}$.

Aortic harvest. Harvests from each group were made at 4 and 16 weeks post-AngII administration. Under isoflurane anesthesia, blood samples were collected through a retrograde left ventricle puncture and stored for biochemical analysis. The viscera and organs were removed, exposing the descending aorta and its branches. Ice cold PBS (500 $\mu \mathrm{l}$ to $1 \mathrm{ml}$ ) was perfused through the aorta through a left ventricular puncture to flush out the aortic lumen via the abdominal aorta bifurcations. The PBS wash was removed from the renal and hepatic bifurcation area using a syringe without a needle. A dissecting microscope was used to magnify the proximal aorta, the aortic arch, the brachiocephalic artery, the left subclavian and left carotid arteries and the thoracic aorta. The entire aortic tree was retrieved through the diaphragm using microforceps and microscissors and stored individually in formalin after adipose and connective tissue was trimmed in ice-cold PBS. The abdominal aorta including the iliac bifurcation was retrieved and stored in the same manner.

Aortic arch and great vessel lesion analysis. Coronal 5- $\mu \mathrm{M}$ sections of the formalin-fixed, paraffin-embedded aortic tree capturing the entire group of aorta ascendens, braciocephalic (innominate) artery, left carotid, left subclavian, aorta descendens and thoracic aorta were collected from all specimens. Three $5-\mu \mathrm{M}$ step sections were taken $50 \mu \mathrm{M}$ apart and then 35 serial sections were taken. Serial sections representative of the brachiocephalic artery lumen throughout each group were selected from 30-35 for Elastin Van Gieson (EVG) staining. Standard EVG staining was performed before mounting slides and capturing digital images with a SPOT digital camera at a magnification of x2. Digital planimetry of a defined area of the aortic tree and its major vessels was performed using Scion Image. A semi-circle of the ascending and descending aortic arch was demarcated by a line drawn from the incision near the aortic root through the opposing 
descending aorta. An arbitrary maximum length of 150 pixels of the brachiocephalic, left carotid and left subclavian artery areas was included with the aortic arch area measurements. Aortic lesions were graded as per Columbia University Core A definitions based on Reddick et al (6) and Kanters et al (17). Type III/Type B aortic dissections as evidenced by subadventitial thrombus and histological confirmation were counted per group. Lesion areas were expressed as the total lesion area over total lumenal area. Necrotic cores were counted in the measured area of each slide.

Abdominal aneurysm analysis. Wet abdominal aortae with and without aneurysms were removed from formalin and placed in ExCell Plus (American Mastertech Scientific, Inc.) non-formalin fixative overnight. Abdominal aortae were trimmed of adipose tissue under ExCell Plus and moved into the physiological orientation in a $60-\mathrm{mm}$ dish of Excell Plus under a dissecting microscope fitted with a SPOT digital camera. A millimeter ruler was placed in frame and images were captured at a magnification of $x 1.5$. The maximal diameter of abdominal aortae and/or their aneurysms was measured against the millimeter ruler in frame to generate diameter values.

Collagen percentage per lesion. Images of arterial trees stained with EVG were loaded as indexed color images utilizing Scion Image software. The curved object pen was used to draw a border around a lesion, which was then placed into a new Scion image file. The area of the lesion was obtained in pixels before repeating the process for the collagenous area of the lesion. The average collagen percentage per lesion was obtained by combining all the lesion collagen areas as a fraction of the combined lesion areas.

Macrophage percentage per lesion and smooth muscle cell percentage per lesion. Images of arterial trees immunohistochemically stained for macrophages or smooth muscle cells were loaded utilizing Scion Image software, using the red channel of the image for analysis. The curved object pen was used to draw a border around a lesion, and the total area of the lesion was obtained in pixels. Using the threshold and/or density function, the color bandwidth representing the brown 3,3'-diaminobenzidine (DAB) signal in the original was demarcated. The pixel area of this selection was determined and recorded. The percentage of macrophages per lesion or of smooth muscle cells (SMC) per lesion was expressed as the sum of the pixel area of the cell type over the sum of the pixel area of the total lesion areas. Primary antibody for the macrophages was Cedarlane Purified Anti-Mouse MAC-2 Monoclonal Antibody Rat IgG2a (CL8942AP) diluted $1: 2,500$ in blocking solution, and secondary antibody was Vector Laboratories Rabbit Biotinylated Anti-Rat IgG $(\mathrm{H}+\mathrm{L})$ affinity purified mouse adsorbed antibody (BA-4001), diluted to $7.5 \mu \mathrm{g} / \mathrm{ml}$ in blocking solution for a working concentration of $15 \mu \mathrm{l}$ of reconstituted antibody $(0.5 \mathrm{mg} / \mathrm{ml}$ in distilled water) into $1 \mathrm{ml}$. The concentration of $7.5 \mu \mathrm{g} / \mathrm{ml}$ corresponds to that of the concentration of Biotinylated secondary antibody used in the Vector Laboratories Vectastain Elite ABC kit for mouse $\operatorname{IgG}$ (PK-6102). The secondary antibody supplied in this kit was BA-2000 at a concentration of $1.5 \mathrm{mg} / \mathrm{ml}$. The working concentration of the secondary antibody was $50 \mu 1$ into $10 \mathrm{ml}$ or $1: 200$ for $7.5 \mu \mathrm{g} / \mathrm{ml}$. For SMC, the primary antibody was Sigma Monoclonal Anti-actin, $\alpha$ smooth muscle clone 1A4, mouse IgG2a isotype (A5228), provided at $2 \mathrm{mg} / 1$ and diluted 1:2,500 in M.O.M. diluent (Vector Laboratories). The secondary antibody used was M.O.M. Biotinylated AntiMouse IgG Reagent. The assays were developed using the Vectastain Elite ABC kit with DAB, which generates a brown color for positive binding.

Lipid percentage per lesion. The assumption was made that, in the complex pathology of the atherosclerotic lesion, the missing spaces found in the necrotic cores represented the free lipid destroyed during formalin-fixed paraffin-embedded section preparation. The size of the lipid area per lesion was expressed as the sum of the pixel areas of the lipid areas over the sum of the pixel area representing the total lesion area.

Plasma lipid assay. Blood for plasma analysis was drawn from the heart. Plasma samples were shipped for rodent blood plasma lipid profile analysis to IDEXX Laboratories (West Sacramento, CA; catalog \#6291).

Ascorbic acid assay. Blood samples were collected from the heart and urine samples from the bladder using syringes. Ascorbic acid content was measured by the fluorescent method detailed by Vislisela et al (18). The data are presented either as $\mu \mathrm{mol} / \mathrm{l}$ for body fluids (blood plasma and urine) or as $\mathrm{nmol} / \mathrm{g}$ wet tissue for tissue (heart and liver homogenates).

Ascorbic acid synthesis assay. The ascorbic acid synthesis rate in collected liver tissue samples was measured by the method detailed by Ching et al (19). Data are presented either as $\mu \mathrm{mol}$ ascorbic acid per gram of wet liver tissue per $30 \mathrm{~min}$, or as mg of ascorbic acid/animal/day after adjustment for the whole liver weight and time.

Statistical analysis. Results are expressed as the means \pm SD for the groups. The independent sample t-test was used to compare the results from the two AngII-treated groups utilizing MedCalc (Mariakerke, Belgium).

\section{Results}

Effect of NM supplementation on AngII-induced aortic arch and great vessel plaque formation. Mean lesion size in the aortas of AngII-treated mice fed the regular rodent diet (Group 3) was 20\% larger $(\mathrm{p}=0.09)$ than that found in NM-supplemented mice (Group 5), as shown in Fig. 1A. Lesion size in untreated ApoE KO mice (Group 2) was 23\% less than that in AngII-treated ApoE KO mice on the control diet, but did not reach statistical significance. Wild-type mice did not develop any lesions. AngII treatment of ApoE KO mice led to a $200 \%(p=0.005)$ increase in lesion severity (Average Columbia core grade) compared to untreated control mice, as shown in Fig. 1B. NM supplementation prior to AngII treatment resulted in a $31 \%(\mathrm{p}=0.13)$ reduction in lesion severity. Likewise, among AngII-treated mice, the average number of necrotic cores per arterial tree section was $39 \%(p=0.35)$ lower in NM-supplemented mice than in unsupplemented 

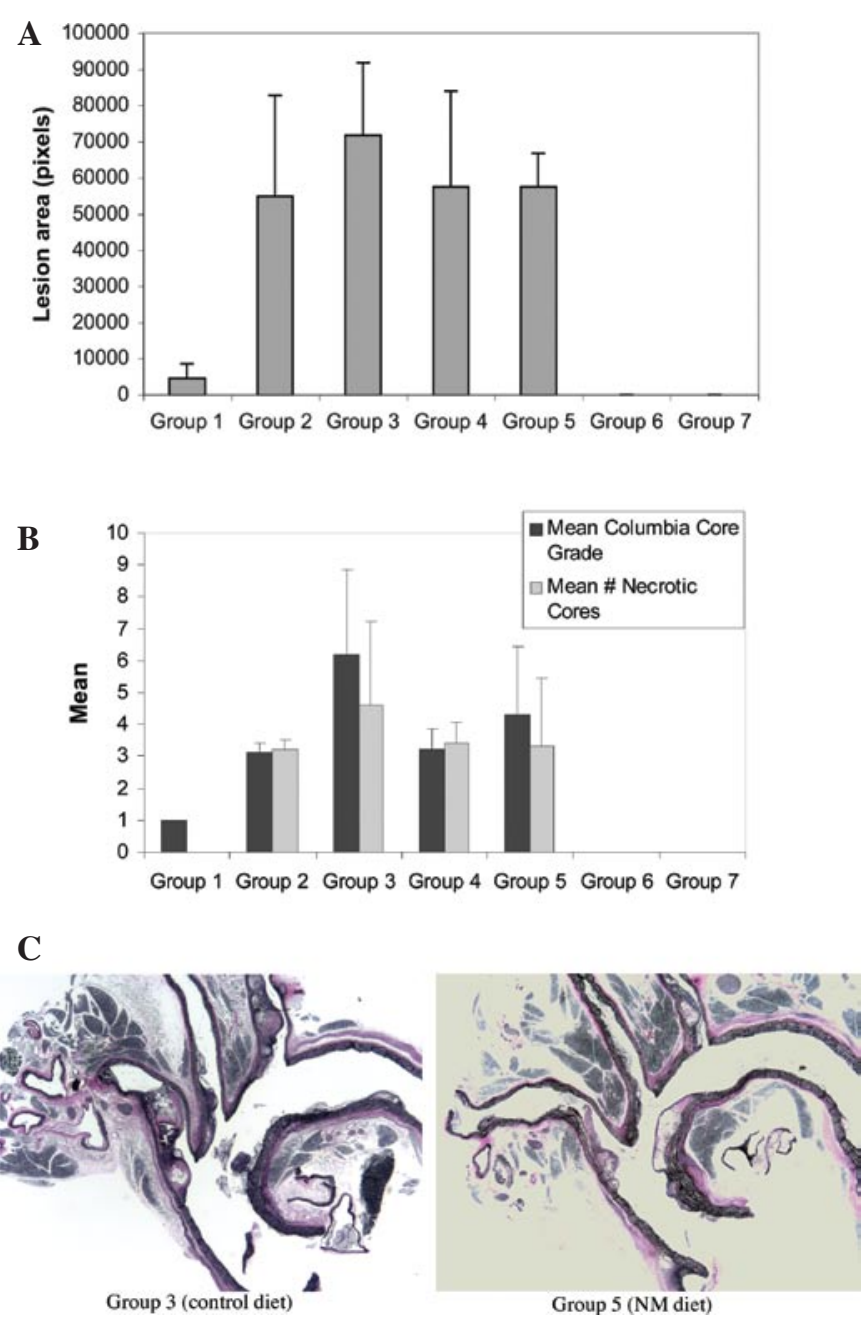

Figure 1. Development of atherosclerotic lesions in the arterial trees of the groups. (A) Lesion differences among the groups. (B) Necrotic cores and lesion grades of the groups. (C) Aortic tree sections representative of atherosclerotic lesions in AngII-treated ApoE KO mice at 4 weeks post AngII administration.

mice, as shown in Fig. 1B. The mean number of necrotic cores in untreated control mice was $30 \%(\mathrm{p}=0.25)$ of that found in AngII-treated controls. Aortic tree sections representative of atherosclerotic lesions in AngII-treated mice are shown in Fig. 1C.

Effect of NM supplementation on the density of macrophages in lesions. The mean density of macrophages in the aortic lesions of AngII-treated mice fed the regular rodent diet (Group 3) was $20 \%$ higher ( $\mathrm{p}=0.033$ ) than that in AngIItreated mice supplemented with NM (Group 5), as shown in Fig. 2A. Mean macrophage infiltration (\% of lesion) in untreated ApoE KO mice (Group 2) was 28\% ( $\mathrm{p}=0.043$ ) less than in AngII-treated ApoE KO mice on the control diet (Group 3). NM-supplemented untreated ApoE KO mice (Group 4) had the lowest macrophage lesion density of the groups, 15\% lower than the unsupplemented untreated ApoE KO mice (Group 2), but the difference did not reach statistical significance. Aortic tree sections representative of macrophages in the atherosclerotic lesions of AngII-treated mice are shown in Fig. 2B.

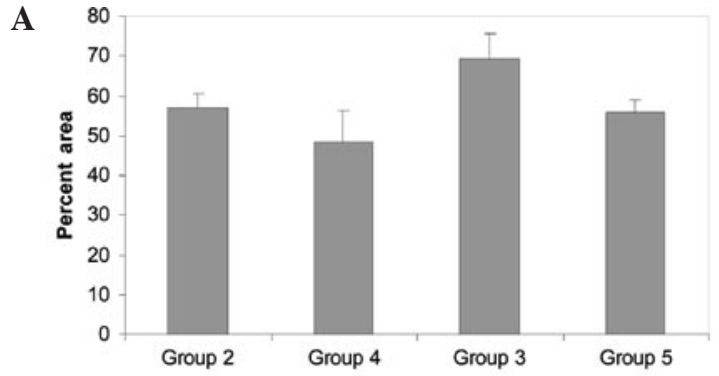

B

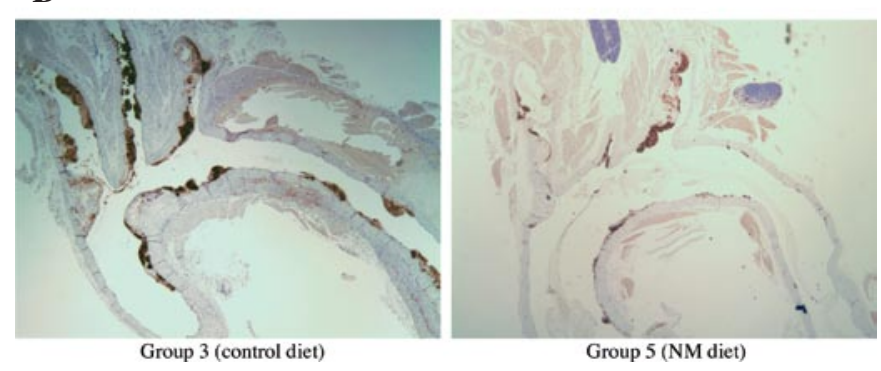

Figure 2. Density of macrophages in atherosclerotic lesions in arterial trees in groups. (A) Mean density of macrophages in groups. (B) Macrophages in representative aortic tree sections of atherosclerotic lesions in AngII-treated ApoE KO mice at 4 weeks post AngII administration.

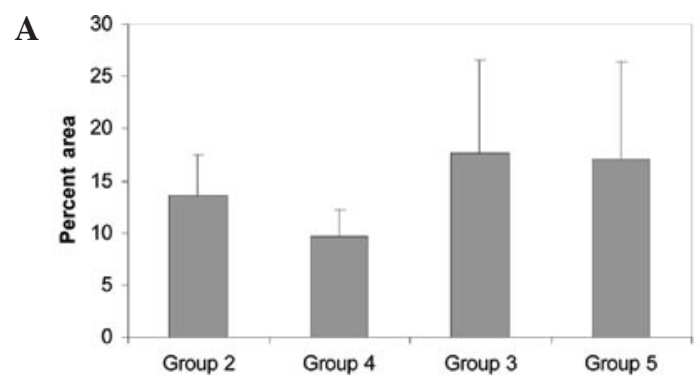

B

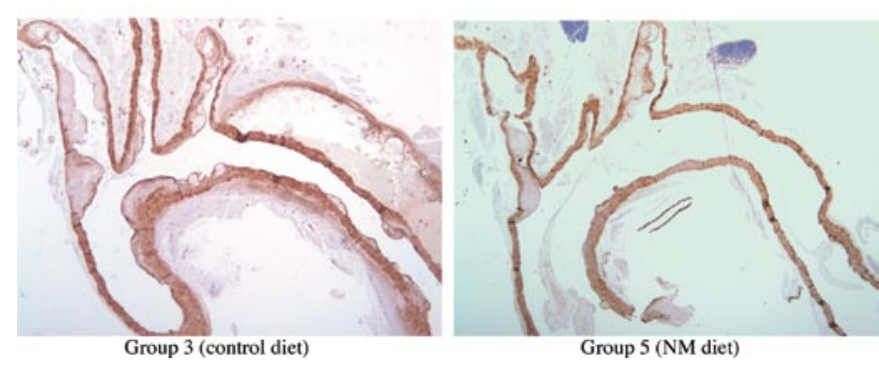

Figure 3. Density of smooth muscle cells in atherosclerotic lesions in the arterial trees of the groups. (A) Mean density of SMC in groups. (B) SMC in representative aortic tree sections of atherosclerotic lesions in AngII-treated ApoE KO mice at 4 weeks post AngII administration.

Effect of NM supplementation on the density of smooth muscle cells in lesions. The mean density of SMC in the aortic lesions of NM-supplemented ApoE KO mice treated with AngII (Group 5) was not significantly different than that found in AngII-treated mice fed the regular rodent diet (Group 3), as shown in Fig. 3A. Mean SMC infiltration 
A

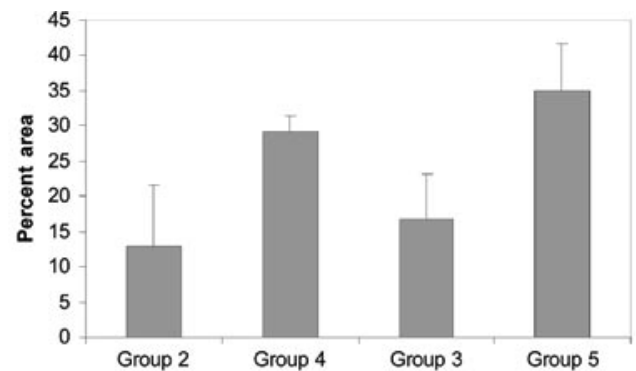

B

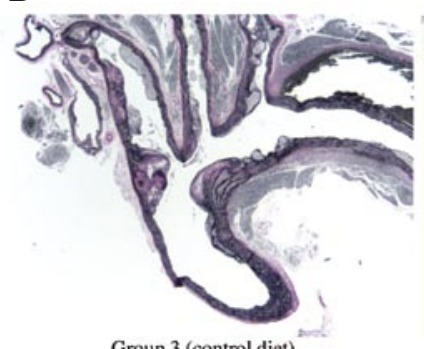

Group 3 (control diet)

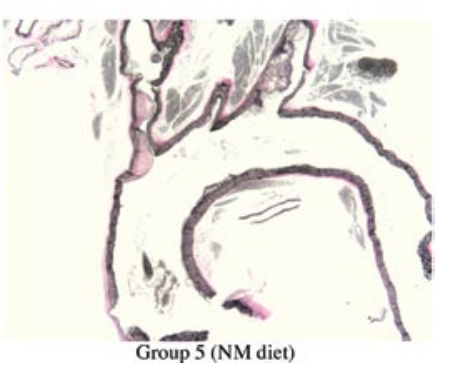

Group 5 (NM diet)

Figure 4. Density of collagen in atherosclerotic lesions in the arterial trees of the groups. (A) Mean density of collagen in the aortic lesions of the groups (B) Collagen in representative aortic tree sections of atherosclerotic lesions in AngII-treated ApoE KO mice at 4 weeks post AngII administration.

(\% of lesion) in untreated ApoE KO mice fed the regular diet (Group 2) was 24\% less than in the lesions of AngII-treated ApoE KO mice fed the regular diet (Group 3), but did not reach statistical significance $(\mathrm{p}=0.49)$. The mean $\mathrm{SMC}$ density of NM-supplemented untreated mice (Group 4) was $29 \%$ less than that in the untreated mice fed the regular diet (Group 2), but did not reach statistical significance $(p=0.23)$. Aortic tree sections representative of SMC in the atherosclerotic lesions of AngII-treated mice are shown in Fig. 3B.

Effect of NM supplementation on the density of collagen in lesions. The mean collagen density (\% of lesion) in the aortic lesions of untreated ApoE $\mathrm{KO}$ mice fed the regular rodent diet (Group 2) was 129\% ( $\mathrm{p}=0.57$ ) of the density in the lesions of AngII-treated ApoE KO mice fed the regular diet (Group 3), as shown in Fig. 4A. The mean collagen density in NM-supplemented ApoE KO mice treated with AngII (Group 5) was 208\% ( $\mathrm{p}=0.026)$ that in the AngII-treated mice fed the regular diet (Group 3). The mean collagen density in NM-supplemented untreated ApoE KO mice (Group 4) was $224 \%(\mathrm{p}=0.034)$ that in the untreated ApoE KO mice fed the regular diet (Group 2). Representative sections of the aortic tree showing collagen density in the lesions of AngII-treated mice are shown in Fig. 4B.

Effect of NM supplementation on AngII-induced Type B aortic dissection. After 4 weeks of AngII administration to ApoE KO mice (Group 3), dramatic Type B aortic dissections occurred, complete with massive sub-adventitial thrombi and false lumens. Thoracic dissections, mostly of Type B, occurred with a frequency of $55.6 \%$ in the animals fed the regular rodent diet (Group 3); however, NM-supplemented AngII-treated ApoE KO mice (Group 5) were found to have a

A

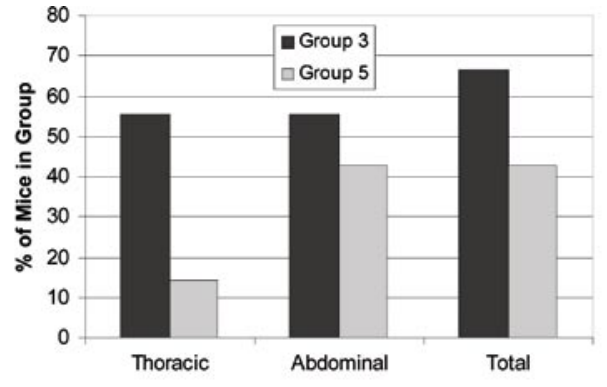

B
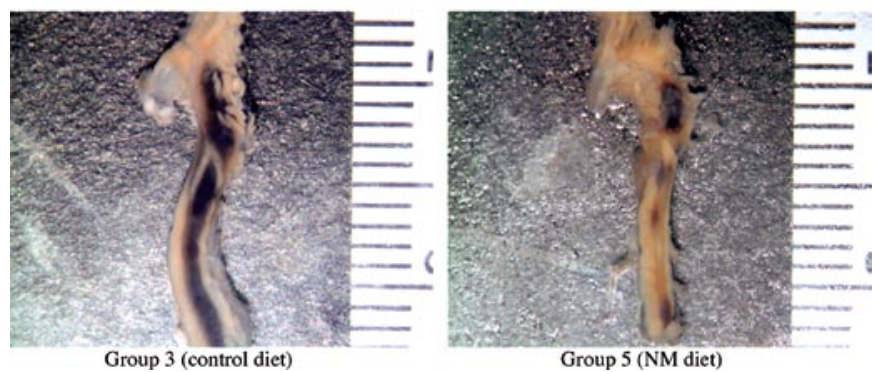

Figure 5. Development of aortic tree dissections and abdominal aneurysms in groups. (A) Frequency of aortic tree dissection and abdominal aortic aneurysms in AngII-treated ApoE KO mice at 4 weeks post AngII administration. (B) Representative aortic dissections from AngII-treated ApoE KO mice.

A

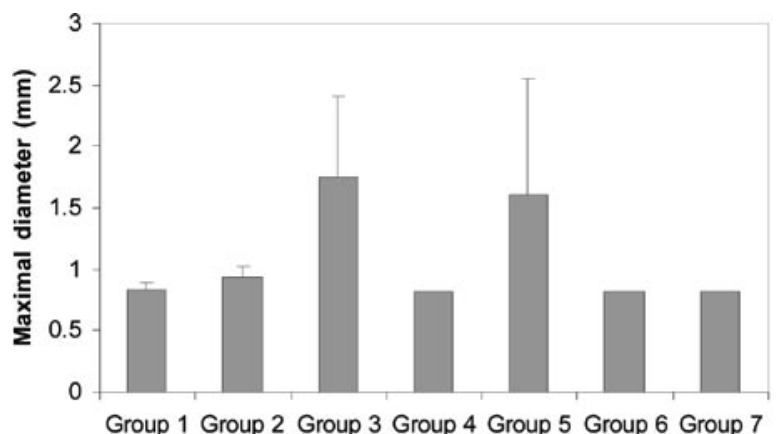

B
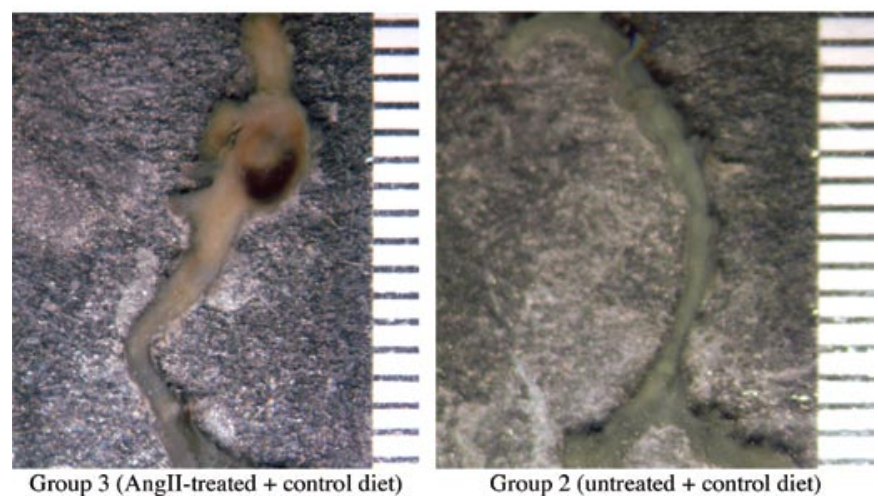

Figure 6. Abdominal aneurysms in groups. (A) Mean maximal diameter of abdominal aneurysms in groups. (B) Representative aortic dissections from AngII-treated ApoE KO mice.

frequency of only $14.3 \%$, as shown in Fig. 5A. Representative sections of the aortic tree from Groups 3 and 5 are shown in Fig. 5B. 


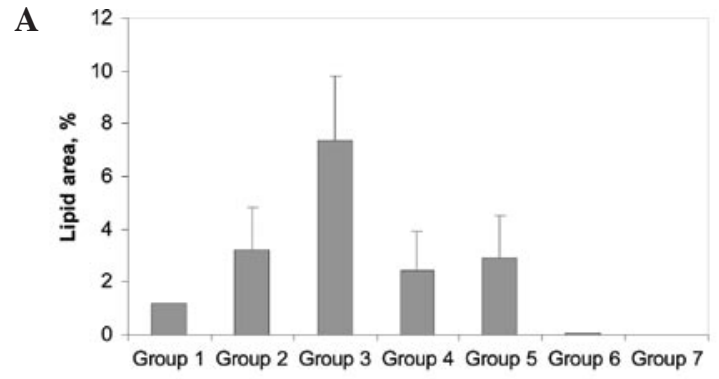

B
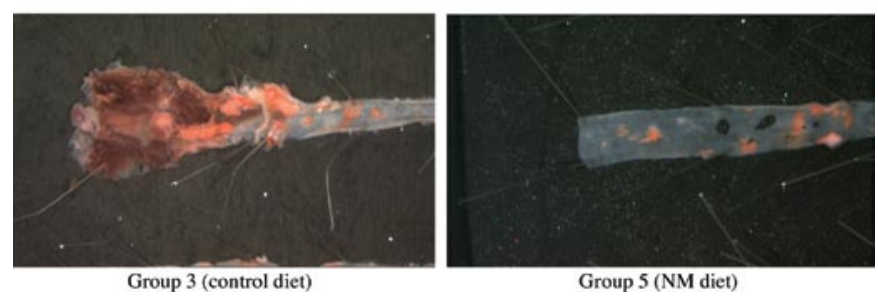

Figure 7. Abdominal aorta lipid area. (A) Mean lipid area percentage per lesion in groups. (B) Representative lipids in AngII-treated ApoE KO mice.

Effect of NM on the size and frequency of AngII-induced abdominal aneurysms. After 4 weeks of AngII administration to ApoE KO mice, mice on the regular rodent diet showed a frequency of abdominal aneurysms of $55.6 \%$, while those on the NM-supplemented diet showed a frequency of $42.9 \%$, as shown in Fig. 5A. AngII treatment of ApoE KO mice maintained on the regular rodent diet (Group 3) resulted in a mean vessel diameter of $1.75 \pm 0.66 \mathrm{~mm}$. As shown in Fig. 6A, NM supplementation of ApoE KO mice treated with AngII reduced the effect of AngII treatment by $8 \%(\mathrm{p}=0.74)$, resulting in a mean diameter of $1.61 \pm 0.95 \mathrm{~mm}$. The mean diameter of abdominal aortas in AngII-treated ApoE KO mice fed a control diet (Group 3) was observed to be $186 \%(p=0.004)$ that in untreated ApoE KO mice (Group 2). Representative abdominal aneurysms found in ApoE $\mathrm{KO}$ groups are shown in Fig. 6B.

Effect of NM supplementation on lipid area in abdominal aortas. The abdominal aorta lipid area in untreated ApoE KO mice increased by $269 \%(\mathrm{p}=0.0015)$ with age (Group 2 vs. Group 1), as shown in Fig. 7A. AngII treatment of ApoE KO mice (Group 3) increased the lipid area by $229 \%$ compared to age-matched untreated ApoE KO mice (Group 2). NM supplementation of AngII-treated ApoE KO mice (Group 5) reduced the lipid area by $60 \%(\mathrm{p}=0.0003)$ compared to AngIItreated mice fed a control diet (Group 3). No lesions were detected in wild-type mice. Representative abdominal aortas stained for lipids from AngII-treated mice (Groups 3 and 5) are shown in Fig. 7B.

Effect of NM supplementation on plasma lipid status. Wildtype mice (Groups 6 and 7) had the lowest total cholesterol and LDL plasma levels and the highest HDL levels of the mouse groups. Untreated 24-week-old ApoE KO mice fed the control diet (Group 1) showed dramatically increased total cholesterol $(869 \%, \mathrm{p}=<0.0001)$ and LDL $(545 \%, \mathrm{p}<0.0001)$ and decreased HDL (65\%, p=0.0003) compared to 24-week-old wild-type mice. Similarly, untreated 36-week-old ApoE KO mice fed the regular diet (Group 2) showed dramatically increased total cholesterol (1020\%, p<0.0001) and LDL (1085\%, p<0.0001), and decreased HDL (49\%, p<0.0001) compared to the age-matched wild-type mice (Group 7). These changes increased with age; 36-week-old ApoE KO mice (Group 2) compared to 24-week ApoE KO mice (Group 1) showed a $133 \%$ increase $(\mathrm{p}<0.0001)$ in total cholesterol, a $226 \%$ increase $(\mathrm{p}=0.0015)$ in $\mathrm{LDL}$ and a decrease to $86 \%(\mathrm{p}=0.27)$ in HDL levels. AngII-treated ApoE KO mice on the regular rodent diet (Group 3) showed an insignificant change in total cholesterol, LDL and HDL compared to untreated ApoE mice (Group 2). Compared to AngII-treated mice on the regular diet (Group 3), NM-supplemented mice treated with AngII showed a significant reduction in total cholesterol (32\% decrease, $\mathrm{p}=0.0001)$ and LDL $(66 \%$ decrease, $\mathrm{p}<0.0001)$ and insignificant reduction in HDL (14\% decrease, $\mathrm{p}=0.12)$. Untreated ApoE KO mice supplemented with NM (Group 4) showed a $27 \%$ decrease $(\mathrm{P}<0.05)$ in LDL levels and no significant differences in total and HDL cholesterol compared to untreated controls (Group 2). Results are shown in Table II.

Effect of NM supplementation on ascorbate status. The ascorbic acid status of the groups is shown in Table III.

Blood plasma ascorbate. The mean plasma ascorbate was significantly increased $(150 \%, \mathrm{p}=0.007)$ in NM-supplemented ApoE KO mice not treated with AngII (Group 4 compared to Group 2). AngII treatment alone resulted in significantly increased $(139 \%, \mathrm{p}=0.007)$ mean plasma ascorbate (Group 3 compared to Group 2). Plasma ascorbate in NM-supplemented AngII-treated mice (Group 5) did not differ from the levels in AngII-treated mice on the control diet (Group 3).

Urine ascorbate. The mean urine ascorbate level in AngIItreated ApoE KO mice supplemented with NM (Group 5) was significantly higher $(200 \%, \mathrm{p}=0.002)$ than in AngII-treated mice on the control diet (Group 3). AngII treatment significantly reduced $(50 \%, \mathrm{p}=0.0001)$ urine ascorbate (Group 3 compared to Group 2). ApoE KO mice (Group 2) had higher (119\%) urine ascorbate compared to age-matched wild-type mice (Group 7), but the difference did not reach statistical significance.

Heart ascorbate. The mean ascorbate level of heart tissue was not affected by NM supplementation (Groups 4 and 5 compared to Groups 2 and 3, respectively). Mice challenged with AngII had a significantly increased heart tissue ascorbate: a $130 \%(\mathrm{p}=0.002)$ increase in Group 3 levels compared to Group 2, and a 104\% $(\mathrm{p}=0.015)$ increase in Group 5 compared to Group 4.

Liver ascorbate. The mean liver tissue ascorbate level in mice supplemented with NM but not challenged with AngII (Group 4) was significantly increased (112\%, p=0.028) compared to the levels observed in untreated mice on the control diet (Group 2). AngII challenge significantly increased (125\%, $\mathrm{p}=0.0004$ ) liver ascorbate (Group 3) compared to untreated mice (Group 2). NM supplementation in mice challenged with AngII (Group 5) did not significantly affect liver ascorbate levels compared to mice on the control diet (Group 3).

Effect of NM supplementation on ascorbate synthesis in the liver. Differences in ascorbate synthesis between KO mice and wild-type mice were not consistent (comparative results are shown in Table IV). Ascorbate synthesis was higher in $\mathrm{KO}$ mice at 24 weeks of age (Group 1 vs. Group 6), but 
Table II. Effect of AngII and NM on plasma cholesterol in ApoE KO and wild-type mice.

\begin{tabular}{lccr}
\hline Groups & Total cholesterol $(\mathrm{mg} / \mathrm{dl})$ & HDL $(\mathrm{mg} / \mathrm{dl})$ & LDL $(\mathrm{mg} / \mathrm{dl})$ \\
\hline $1(\mathrm{n}=9)$ & $603.8 \pm 83.9^{\mathrm{a}}$ & $31.0 \pm 9.6^{\mathrm{a}}$ & $39.8 \pm 11.5^{\mathrm{a}}$ \\
$2(\mathrm{n}=9)$ & $800.8 \pm 187.2^{\mathrm{b}}$ & $26.8 \pm 5.6^{\mathrm{a}, \mathrm{b}}$ & $90.1 \pm 37.9^{\mathrm{b}}$ \\
$3(\mathrm{n}=9)$ & $768.5 \pm 72.9^{\mathrm{b}}$ & $29.3 \pm 5.8^{\mathrm{a}}$ & $87.0 \pm 14.0^{\mathrm{b}}$ \\
$4(\mathrm{n}=9)$ & $780.1 \pm 111.8^{\mathrm{b}}$ & $22.7 \pm 6.2^{\mathrm{b}}$ & $57.2 \pm 18.5^{\mathrm{c}}$ \\
$5(\mathrm{n}=7)$ & $524.4 \pm 100.1^{\mathrm{a}}$ & $25.3 \pm 3.0^{\mathrm{a}, \mathrm{b}}$ & $30.3 \pm 10.0^{\mathrm{a}}$ \\
$6(\mathrm{n}=8)$ & $69.5 \pm 4.5^{\mathrm{c}}$ & $48.0 \pm 3.5^{\mathrm{c}}$ & $7.3 \pm 0.6^{\mathrm{d}}$ \\
$7(\mathrm{n}=9)$ & $78.5 \pm 3.7^{\mathrm{c}}$ & $55.1 \pm 3.0^{\mathrm{c}}$ & $8.3 \pm 0.7^{\mathrm{d}}$ \\
\hline
\end{tabular}

Superscripts indicate statistically significant differences between groups.

Table III. Ascorbic acid levels in wild-type and ApoE KO mice.

\begin{tabular}{lcccc}
\hline Groups & $\begin{array}{c}\text { Plasma ascorbate } \\
(\mu \mathrm{mol} / \mathrm{l})\end{array}$ & $\begin{array}{c}\text { Heart tissue ascorbate } \\
(\mathrm{nmol} / \mathrm{g})\end{array}$ & $\begin{array}{c}\text { Liver tissue ascorbate } \\
(\mathrm{nmol} / \mathrm{g})\end{array}$ & $\begin{array}{c}\text { Urine ascorbate } \\
(\mathrm{mmol} / \mathrm{l})\end{array}$ \\
\hline $1(\mathrm{n}=9)$ & $70.8 \pm 16.6^{\mathrm{a}, \mathrm{c}}$ & $446.2 \pm 43.8^{\mathrm{b}, \mathrm{c}}$ & $982.3 \pm 188.7^{\mathrm{a}, \mathrm{b}, \mathrm{c}}$ & $\mathrm{ND}$ \\
$2(\mathrm{n}=9)$ & $70.0 \pm 16.7^{\mathrm{a}, \mathrm{c}}$ & $394.2 \pm 39.1^{\mathrm{a}}$ & $867.7 \pm 108.6^{\mathrm{a}}$ & $2.30 \pm 0.52^{\mathrm{b}}$ \\
$3(\mathrm{n}=9)$ & $97.4 \pm 20.3^{\mathrm{b}}$ & $515.9 \pm 91.0^{\mathrm{b}}$ & $1087.9 \pm 101.2^{\mathrm{b}}$ & $1.18 \pm 0.73^{\mathrm{a}}$ \\
$4(\mathrm{n}=9)$ & $104.7 \pm 29.2^{\mathrm{b}}$ & $406.6 \pm 47.2^{\mathrm{a}, \mathrm{c}}$ & $974.3 \pm 76.4^{\mathrm{c}}$ & $3.01 \pm 0.44^{\mathrm{c}}$ \\
$5(\mathrm{n}=7)$ & $86.6 \pm 16.8^{\mathrm{b}, \mathrm{c}}$ & $469.7 \pm 43.8^{\mathrm{b}}$ & $1014.1 \pm 178.9^{\mathrm{a}, \mathrm{b}, \mathrm{c}}$ & $2.19 \pm 0.75^{\mathrm{b}, \mathrm{d}}$ \\
$6(\mathrm{n}=8)$ & $65.3 \pm 6.4^{\mathrm{a}}$ & $380.1 \pm 54.8^{\mathrm{a}}$ & $882.5 \pm 140.9^{\mathrm{a}, \mathrm{c}}$ & $\mathrm{ND}$ \\
$7(\mathrm{n}=9)$ & $57.4 \pm 10.9^{\mathrm{a}}$ & $381.3 \pm 43.0^{\mathrm{a}}$ & $1027.7 \pm 131.7^{\mathrm{b}, \mathrm{c}}$ & $1.56 \pm 0.78^{\mathrm{a}, \mathrm{d}}$ \\
\hline
\end{tabular}

Superscripts indicate statistically significant differences between groups.

Table IV. Ascorbic acid synthesis in wild-type and ApoE KO mice.

\begin{tabular}{|c|c|c|c|}
\hline Groups & $\begin{array}{c}\text { Ascorbate synthesis rate } \\
(\mu \mathrm{mol} / \mathrm{g} \text { wet liver tissue/30 min) }\end{array}$ & Liver weight (mg) & $\begin{array}{l}\text { Ascorbate synthesis rate } \\
\text { (mg per animal per } 24 \mathrm{~h} \text { ) }\end{array}$ \\
\hline $1(n=9)$ & $1.99 \pm 0.22^{\mathrm{a}, \mathrm{c}}$ & $1357.2 \pm 108.1$ & $24.59 \pm 0.93^{\mathrm{b}}$ \\
\hline $2(n=9)$ & $1.35 \pm 0.22^{\mathrm{a}, \mathrm{c}}$ & $1306.3 \pm 86.9$ & $14.94 \pm 2.67^{\mathrm{c}}$ \\
\hline $3(n=9)$ & $1.40 \pm 0.12^{\mathrm{b}}$ & $1418.4 \pm 129.6$ & $16.24 \pm 0.96^{c}$ \\
\hline $4(n=9)$ & $1.42 \pm 0.14^{\mathrm{b}}$ & $1411.2 \pm 94.0$ & $16.97 \pm 2.43^{\mathrm{a}, \mathrm{c}}$ \\
\hline $5(n=7)$ & $1.45 \pm 0.15^{\mathrm{b}, \mathrm{c}}$ & $1572.0 \pm 146.3$ & $20.43 \pm 2.43^{\mathrm{d}}$ \\
\hline $6(n=8)$ & $1.27 \pm 0.43^{\mathrm{a}}$ & $1271.7 \pm 153.4$ & $14.89 \pm 5.01^{\mathrm{a}, \mathrm{c}}$ \\
\hline $7(n=9)$ & $1.65 \pm 0.27^{\mathrm{a}}$ & $1411.3 \pm 71.1$ & $19.69 \pm 3.38^{\mathrm{a}, \mathrm{d}}$ \\
\hline
\end{tabular}

Superscripts indicate statistically significant differences between groups.

lower at 36 weeks (Group 2 vs. Group 7). AngII challenge did not influence ascorbate synthesis (Group 3 vs. Group 2). The supplementation with NM of diets fed to mice caused an increase in ascorbate synthesis in the liver (Groups 4 and 5 vs. Groups 2 and 3, respectively). However, this increase only reached statistical significance in AngII-challenged animals (Group 5 vs. Group 3). AngII challenge did not significantly affect the ascorbate synthesis rate. Ascorbate daily synthesis (calculated per whole liver for each animal for a 24-h period) was in the range of 10-30 mg ascorbate, whereas ascorbate supplementation in this study was $<4 \mathrm{mg}$ a day per animal, based on food consumption of $4 \mathrm{~g}$ per animal per day.

Correlations between ascorbate levels. Plasma ascorbate levels were positively correlated to urine ascorbate levels (correlation coefficient $\mathrm{r}=0.2385, \mathrm{p}=0.067$ ), probably indicating the increasing clearance of excess ascorbate. Wet heart tissue ascorbate positively correlated to plasma ascorbate (correlation coefficient $r=0.3170, p=0.014$ ), probably indicating its dependence on supply. Wet liver tissue did not correlate to plasma 
ascorbate, probably indicating impact from ascorbate biosynthesis. Liver tissue ascorbate levels were positively correlated to heart ascorbate levels (correlation coefficient $r=0.4283$, $\mathrm{p}=0.0006$ ). Liver tissue ascorbate was negatively correlated to urine ascorbate (correlation coefficient $\mathrm{r}=-0.4152, \mathrm{p}=0.0069$ ), indicating a reverse relationship between ascorbate accumulation in liver tissue depots and ascorbate clearance. However, ascorbate biosynthesis did not correlate to any tissue ascorbate level tested.

\section{Discussion}

Excess AngII has been implicated in the acceleration of pathological processes that lead to the development of atherosclerosis and vascular diseases such as inflammation through the induction of cytokines and matrix remodeling, secondary to the modulation of collagen synthesis and matrix metalloproteinases (5). The major objective of this study was to explore the effect of nutrient supplementation on AngII-mediated great vessel atherosclerosis in ApoE KO mice, examining the various parameters associated with the pathogenesis of atherosclerosis in the ApoE KO groups and comparing the effect of nutrient supplementation to a normal diet on these parameters, to elucidate the mechanisms involved. AngII-mediated injuries, which included increases in atherosclerotic lesion size (by 31\%) and severity (by 200\%) as well as an increase in the number of necrotic cores (by 44\%) were attenuated by nutrient supplementation. NM reduced lesion size in AngII-treated mice by $20 \%$ and reduced lesion severity by $31 \%$ compared to AngIItreated mice fed the regular rodent diet. Likewise, among the AngII-treated mice, the average number of necrotic cores per arterial tree section was $39 \%$ higher in unsupplemented mice than in NM-supplemented mice.

Furthermore, the frequency of thoracic aortic dissections and abdominal aortic aneurysms induced by AngII treatment in ApoE KO mice was decreased with NM supplementation. Abdominal aortic dissections, a major cause of mortality in the elderly, are the permanent dilations of the artery (normally defined as an increase in diameter of $>50 \%$ ), characterized by the degeneration of medial elastic fibers, thinning of media, adventitial hypertrophy and the accumulation of macrophages and $\mathrm{T}$ and $\mathrm{B}$ lymphocytes, atherosclerotic lesions and thrombi (20). Saraff (21) noted that in mice infused with AngII, abdominal aortic aneurysms were formed prior to evidence of atherosclerotic lesions. After 1-4 days of AngII treatment, mice showed macrophage accumulation on the adventitia of suprarenal aortas and in the thoracic aortic region and, after 4-10 days, vascular hematomas (21). In our study, the mean density of macrophages in the aortic lesions of AngII-treated mice fed the regular rodent diet was $20 \%$ higher than in AngII-treated mice supplemented with NM.

Increasing evidence indicates that plaque stabilization is attributable to the composition of atherosclerotic plaques, and that inflammation plays an important role in the formation and progress of vulnerable atheorsclerotic plaques, which are prone to rupture (22-24). Increased lipid content, especially cholesterol ester, has been associated with the decreased mechanical strength of the plaques $(22,24)$. Additionally, strong evidence suggests that the oxidation of low-density lipoprotein by free radicals is one of the most important factors for the initiation of atherosclerosis $(25,26)$. Compared to AngII-treated mice on the regular diet, NM-supplemented mice challenged with AngII showed a significant reduction in total cholesterol (32\% decrease, $\mathrm{p}=0.0001)$ and LDL $(66 \%$ decrease, $\mathrm{p}<0.0001)$. Furthermore, increased collagen content in the plaques, especially the fibrous cap, maintains plaque stability and an intact fibrous cap (22). The ratio of lipids to collagen content is proposed as an important index to evaluate plaque stability (24). In our study, mean collagen density in NM-supplemented ApoE KO mice treated with AngII was 208\% that in the AngII-treated mice fed the regular diet.

Atherosclerotic plaque vulnerability has also been attributed to MMPs, which are responsible for the degradation of the extracellular matrix (ECM), and has been linked to the inflammatory and fibrinolytic system, as well as to lipid and homocysteine levels (27). The composition and structure of the ECM undergoes significant changes during the atherosclerotic process as a result of the compromised regulation of the matrix metalloproteinase network and modifications of matrix component synthesis and deposition. An immunochemical analysis by Katsuda et al (28) demonstrated an increased distribution of types I and III collagen in the thickened intima of all stages of atherosclerotic lesions. Collagen types I and III, which are missing in normal intima, play a critical role in the formation and progress of atherosclerosis. Though collagen IV, a basement membrane collagen present in normal intima, showed some increase with the progression of atherosclerosis, the relative amount of collagen IV to collagen I decreased significantly in atherosclerotic tissues (28). MMP-9 degrades collagen IV and $\mathrm{V}$, as well as fibronectin. In a previous in vitro study, increased strength and integrity of the ECM compared to the control was produced by smooth muscle cells (SMC) exposed to a mixture of nutrients (29). The resultant ECM was characterized by an increased collagen type IV to I ratio and profound inhibitory effects on aortic SMC migration and connective tissue invasion (29). We also found that the NM showed the potential to block the development of atherosclerotic lesions by the inhibition of atherogenic responses of vascular SMC to pathological stimuli, such as aortic SMC proliferation, invasion and migration from the vessel medial to intimal layer and secretion of inflammatory cytokines (30). In a subsequent study, we found that plantderived micronutrients suppressed monocyte adhesion to a cultured human aortic endothelial cell layer by modulating its extracellular matrix composition (31). Aortic endothelial cell exposure to micronutrients reduced the capacity of the ECM to bind monocyes in a dose-dependent manner. This effect was accompanied by profound changes in the ECM composition. Correlation analysis revealed that changes in monocyte adhesion had the strongest positive correlation with ECM content for laminin, fibronectin, collagen types II, I and IV, biglycen, heparan sulfate and elastin (31).

Vitamin C plays a crucial role in connective tissue metabolism, acting as the reducing co-factor in the reaction catalyzed by prolyl and lysyl hydroxylases. Hydroxyproline and hydroxylysine are essential for the secretion, cross-linking and maturation of collagen, a key component of extracellular matrix important for the maintenance of vascular integrity $(32,33)$. Thus, we decided to determine the ascorbate status in all the mouse groups in order to detect any variations. In 
our study, mean plasma ascorbate and heart and liver tissue ascorbate were increased in AngII-treated ApoE KO mice compared to untreated mice, indicating increased synthesis of ascorbate in response to AngII-induced atheorsclerotic damage. NM-supplemented AngII-treated mice showed slightly lower levels than the AngII-treated mice fed a control diet. By contrast, in ApoE $\mathrm{KO}$ mice unable to synthesize vitamin $\mathrm{C}\left(\mathrm{Gulo}^{-}\right.$- ApoE $\left./\right)$, Nakata and Maeda showed that chronic vitamin $\mathrm{C}$ deficiency severely compromised collagen deposition and induced a type of plaque morphology potentially vulnerable to rupture (34). Though lesion size was not affected by vitamin $\mathrm{C}$ status, collagen was significantly reduced and necrotic cores were larger in vitamin C-deficient mice (34).

In conclusion, NM consumption prior to AngII administration in ApoE (-/-) mice minimized atherosclerotic associated pathologies, thoracic aortic dissections and abdominal aneurysms. Furthermore, mechanistically, supplementation decreased inflammatory monocyte and SMC recruitment to the plaques and increased collagen content, thus increasing the stability of the plaques. In addition, NM supplementation of mice resulted in a significant reduction in total cholesterol and low-density lipoprotein.

\section{Acknowledgements}

This study was funded by the Dr. Rath Health Foundation (Plantation, FL), a non-profit organization.

\section{References}

1. Lithell H: Pathogenesis and prevalence of atherosclerosis in hypertensive patients. Am J Hypertens 7: 28-68, 1994.

2. Alexander RW: Hypertension and pathogenesis of atherosclerosis: oxidative stress and the mediation of arterial inflammatory response. Hypertension 25: 155-161, 1995.

3. Zadelaar S, Kleeman R, Verschuren L, De Vries-van der Weji J, van der Hoorn J, Princen HM and Kooistra T: Mouse models for atherosclerosis and pharmaceutical modifiers. Artheroscler Thromb Vasc Biol 27: 1706-1721, 2007.

4. Whitman SC: A practical approach using mice in atherosclerosis research. Clin Biochem Rev 25: 81-93, 2004.

5. Ayabe N, Babaev VR, Tang Y, Tanizawa T, Fogo AB, Linton MF, Ichikawa Y, Fazio $S$ and Kon V: Transiently heightened angiotensin II has distinct effects on atherosclerosis and aneurysm formation in hyperlipidemic mice. Atherosclerosis 184: 312-321, 2006.

6. Reddick RL, Zhang SH and Maeda N: Atherosclerosis in mice lacking apoE. Evaluation of lesional development and progression. Atheroscler Thromb 14: 141-147, 1994.

7. Ness AR and Powles JW: Fruit and vegetables and cardiovascular disease: a review. Int J Epidemiol 26: 1-13, 1997.

8. Chopra M, O'Neill ME, Keogh N, Wortley G, Southon S and Thurnham DI: Influence of increased fruit and vegetable intake on plasma and lipoprotein caroteniods and LDL oxidation in smokers and nonsmokers. Clin Chem 46: 1818-1829, 2000.

9. Esposito K, Nappo F, Giugliano F, Giugliano G, Marfella R and Giugliano D: Effect of dietary antioxidants on postprandial endothelial dysfunction induced by a high-fat meal in healthy subjects. Am J Clin Nutr 77: 139-143, 2003.

10. Dragsted LO, Krath B, Ravn-Haren G, Vogel UB, Vinggaard AM, Jensen PB, Loft S, Rasmussen SE, Sandstrom B and Pedersen A: Biological effects of fruit and vegetables. Proc Nutr Soc 65: 61-67, 2006.

11. Silaste ML, Rantala M, Alfthan G, Aro A and Kesaniemi YA: Plasma homocysteine concentration is decreased by dietary intervention. Br J Nutr 89: 295-301, 2003.

12. Kneckt P, Jarvinen R, Reunanen A and Maaatela J: Flavonoid intake and coronary mortality in Finland: a cohort study. BMJ 312: 478-481, 1996

13. Riemersma RA, Wood DA, Macintyre CCA, Elton RA, Gey KF and Oliver MF: Risk of angina pectoris and plasma concentrations of vitamin A, C and E and carotene. Lancet 337: 1-5, 1991.
14. Hodis HN, Mack WJ, La Bree L, Selzer RH, Liu CR and Azen SP: Serial coronary angiographic evidence that antioxidant vitamin intake reduces progression of coronary artery atherosclerosis. JAMA 275: 1893-1896, 1996.

15. Rath $\mathrm{M}$ and Niedzwiecki A: Nutritional supplement halts progression of early coronary atherosclerosis. J Appl Nutr 48: 67-78, 1996.

16. Miura Y, Chiba T, Tomia I, Koizumi H, Miura S, Umegaki K, Hara Y, Ikeda M and Tomita T: Tea catechins prevent the development of atherosclerois in apoprotein E-deficient mice. J Nutr 131: 27-32, 2001

17. Kanters E, Pasparakis M, Gijbels, Vergouwe MN, Partouns-Hendricks I, Fijneman RJ, Clausen BE, Förster I, Kockx MM, Rajewsky K, Kraal G, Hofker MH and De Winther MP: Inhibition of NF- $\mathrm{BB}$ activation in macrophages increases atherosclerosis in LDL receptor-deficient mice. J Clin Invest 112: 1176-1185, 2003.

18. Vislisela JM, Schafera FQ and Buettner GR: A simple and sensitive assay for ascorbate using a plate reader. Anal Biochem 365: 31-39, 2007

19. Ching S, Mahan DC, Moreau RR and Dabrowski K: Modification of analytical procedures for determining vitamin $C$ enzyme (L-gulonolactone oxidase) activity in swine liver. J Nutr Biochem 14: 139-146, 2003.

20. Daugherty A and Cassis LA: Mechanisms of abdominal aortic aneurysm formation. Curr Atheroscler Rep 4: 222-227, 2002.

21. Saraff K, Babamusta F, Cassis LA and Daugherty A: Aortic dissection precedes formation of aneurysms and atherosclerosis in angiotensin II-infused, apolipoprotein E-deficient mice. Arterioscler Thromb Vasc Biol 23: 1621-1626, 2003.

22. Nakamura M, Lee DP and Young AC: Identification and treatment of vulnerable plaque. Rev Cardiovasc Med 5 (Suppl 2): 22-33, 2004.

23. Peter L and Massanor A: Stabilization of atherosclerotic plaques. New mechanisms and clinical targets. Nature Med 8: 1257-1262, 2002.

24. Naghavi M, Libby P, Falk E, et al: From vulnerable plaque to vulnerable patient: a call for new definitions and risk assessment strategies: Part I. Circulation 108: 1664-1672, 2003.

25. Witzum JL and Steinberg D: Role of oxidized low density lipoprotein in atherogenesis. J Clin Invest 88: 1785-1792, 1991.

26. Heinecke JW: Oxidants and antioxidants in the pathogenesis of atherosclerosis: implications for the oxidized low density lipoprotein hypothesis. Atherosclerosis 141: 1-15, 1998.

27. Marc F, Mark S, Martin G and Michael H: Matrix metalloproteinases in cerebrovascular diseases. Cerbrovasc Dis 20: 141-151, 2005.

28. Katsuda S, Okada Y, Minamoto T, Oda Y, Matsui Y and Nakanishi I: Collagens in human atherosclerosis. Immunohistochemical analysis using collagen type specific antibodies. Arterioscler Thromb Vasc Biol 12: 494-502, 1992.

29. Ivanov V, Ivanova S, Roomi MW, Kalinovsky T, Niedzwiecki A and Rath M: Extracellular matrix-mediated control aortic smooth muscle cell growth and migration by a combination of ascorbic acid, lysine, proline, and catechins. J Cardiovasc Pharmacol 50: 541-547, 2007.

30. Ivanov V, Roomi MW, Kalinovsky T, Niedzwiecki A and Rath M: Anti-atherogenic effects of a mixture of ascorbic acid, lysine, proline, arginine, cysteine, and green tea phenolics in human aortic smooth muscle cells. J Cardiovasc Pharmacol 49: 140-145, 2007.

31. Ivanov V, Ivanova S, Kalinovsky T, Niedzwiecki A and Rath M: Plant-derived micronutrients suppress monocyte adhesion to cultured human aortic endothelial cell layer by modulating its extracellular matrix composition. J Cardiovasc Pharmacol 52: 55-65, 2008.

32. Rath M: Solution to the puzzle of human cardiovascular disease: its primary cause is ascorbate deficiency, leading to the deposition of lipoprotein(a) and fibrinogen/fibrin in the vascular wall. J Ortho Med 6: 125-134, 1991.

33. Walmsley AR, Batten MR, Lad U and Bulleid NJ: Intracelllular retention of procollagen within the endoplasmic reticulum is mediated by prolyl 4-hydroxylase. J Biol Chem 274: 14884-14892, 1999.

34. Nakata $Y$ and Maeda N: Vulnerable atherosclerotic plaque morphology in apolipoprotein E-deficient mice unable to make ascorbic acid. Circulation 105: 1485-1490, 2002. 